\title{
Limit theorems for delayed sums of random sequence
}

\author{
Ding Fang-qing ${ }^{1}$ and Wang Zhong-zhi ${ }^{2^{*}}$
}

* Correspondence: wzz30@ahut. edu.cn

${ }^{2}$ Faculty of Mathematics \& Physics, AnHui University of Technology, Ma'anshan 243002, P. R. China Full list of author information is available at the end of the article

\section{Abstract}

For a sequence of arbitrarily dependent random variables $\left(X_{n}\right)_{n \in \mathbf{N}}$ and Borel sets $\left(B_{n}\right)$ $n \in \mathbf{N}$, on real line the strong limit theorems, represented by inequalities, i.e. the strong deviation theorems of the delayed average $S_{n . k_{n}}(\omega)$ are investigated by using the notion of asymptotic delayed log-likelihood ratio. The results obtained popularizes the methods proposed by Liu.

Mathematics Subject Classification 2000: Primary, 60F15.

Keywords: strong deviation theorem, likelihood ratio, delayed sums

\section{Introduction}

Let $\left(a_{n}\right)_{n \in \mathbf{N}}$ be a sequence of real numbers and let $\left(k_{n}\right)_{n \in \mathbf{N}}$ be a sequence of positive integers. The numbers

$$
\rho_{n, k_{n}}=\left\{\sum_{j=1}^{k_{n}} a_{n+j-1}\right\} / k_{n}
$$

are called the (forward) delayed first arithmetic means (See [1]). In [2], using the limiting behavior of delayed average, Chow found necessary and sufficient conditions for the Borel summability of i.i.d. random variables and also obtained very simple proofs of a number of well-known results such as the Hsu-Robbins-Spitzer-Katz theorem. In [3], Lai studied the analogues of the law of the iterated logarithim for delayed sums of independent random variables. Recently, Chen [4] has presented an accurate description the limiting behavior of delayed sums under a non-identically distribution setup, and has deduced Chover-type laws of the iterated logarithm for them.

Our aim in this article is to establish strong deviation theorems (limit theorem expressed by inequalities, see [5]) of delayed average for the dependent absolutely continuous random variables. By using the notion of asymptotic delayed log-likelihood ratio, we extend the analytic technique proposed by Liu [5] to the case of delayed sums. The crucial part of the proof is to construct a delayed likelihood ratio depending on a parameter, and then applies the Borel-Cantelli lemma.

Throughout, let $\left(X_{n}\right)_{n \in \mathbf{N}}$ be a sequence of absolutely continuous random variables on a fixed probability space $\{\Omega, \mathcal{F}, P\}$ with the joint density function $g^{1, n}\left(x_{1}, \ldots, x_{n}\right), n \in$ $\mathbf{N}$, and $f_{j}(x), j=1,2, \ldots$ be the the marginal density function of random variable $X_{j}$. $\left(k_{n}\right)$ 
$n \in \mathbf{N}$ be a subsequence of positive integers, such that, for every $\varepsilon>0$, $\sum_{n=1}^{\infty} \exp \left(-k_{n} \varepsilon\right)<\infty$.

Definition 1. The delayed likelihood ratio is defined by

$$
\mathcal{L}_{n}(\omega)= \begin{cases}\frac{\prod_{j=n}^{n+k_{n}-1} f_{j}\left(X_{j}\right)}{g^{n, n+k_{n}-1\left(X_{n}, \ldots, X_{n+k_{n}-1}\right)}}, & \text { if denominator }>0 \\ 0, & \text { otherwise }\end{cases}
$$

Let

$$
\mathcal{L}(\omega)=-\lim _{n} \inf \frac{1}{k_{n}} \log \mathcal{L}_{n}(\omega)
$$

$\mathcal{L}(\omega)$ is called asymptotic delayed log-likelihood ratio, where $g^{n, n+k_{n}-1}\left(x_{n}, \ldots, x_{n+k_{n}-1}\right)$ denotes the joint density function of random vector $\left(X_{n}, \ldots, X_{n+k_{n}-1}\right), \omega$ is a sample point (with $\log 0=-\infty$ ).

It will be shown in Lemma 1 that $\mathcal{L}(\omega) \geq 0$ a.e. in any case.

Remark 1. It will be seen below that $\mathcal{L}(\omega)$ has the analogous properties of the likelihood ratio in [5], Although $\mathcal{L}(\omega)$ is not a proper metric among probability measures, we nevertheless consider it as a measure of "discrimination" between the dependence (their joint distribution) and independence (the product of their marginals). Obviously, $\mathcal{L}_{n}(\omega)=1$, a.e. $n \in \mathbf{N}$ if $\left(X_{n}\right)_{n \in \mathbf{N}}$ is independent. In view of the above discussion of the asymptotic logarithmic delayed likelihood ratio, it is natural for us to think of $\mathcal{L}(\omega)$ as a measure how far (the random deviation) of $\left(X_{n}\right)_{n \in \mathbf{N}}$ is from being independent and how dependent they are. The closer $\mathcal{L}(\omega)$ approaches to 0 , the smaller the deviation is.

Lemma 1. Let $\mathcal{L}_{n}(\omega)$ be define as above, then

$$
\limsup _{n} \frac{1}{k_{n}} \log \mathcal{L}_{n}(\omega) \leq 0 \text {, a.e. }
$$

Proof. Let $B=\left\{\left(x_{n}, \ldots, x_{n+k_{n}-1}\right): g^{n, n+k_{n}-1}\left(x_{n}, \ldots, x_{n+k_{n}-1}\right)>0\right\}$. Since

$$
\begin{aligned}
E\left[\mathcal{L}_{n}(\omega)\right] & \int \ldots \int_{\left(x_{n}, \ldots, x_{n+k_{n}-1}\right) \in B} \frac{\prod_{j=n}^{n+k_{n}-1} f_{j}\left(x_{j}\right)}{g^{n, n+k_{n}-1}\left(x_{n}, \ldots, x_{n+k_{n}-1}\right)} \\
& \cdot g^{n, n+k_{n}-1}\left(x_{n}, \ldots, x_{n+k_{n}-1}\right) d x_{n} \ldots d x_{n+k_{n}-1} \\
= & \int \ldots \int_{\left(x_{n}, \ldots, x_{n+k_{n}-1}\right) \in B} \prod_{j=n}^{n+k_{n}-1} f_{j}\left(x_{j}\right) d x_{n} \ldots d x_{n+k_{n}-1} \\
\leq & \int \ldots \int_{\left(x_{n}, \ldots, x_{n+k_{n}-1}\right) \in \mathbf{R}^{k_{n}}} \prod_{j=n}^{n+k_{n}-1} f_{j}\left(x_{j}\right) d x_{n} \ldots d x_{n+k_{n}-1}=1 .
\end{aligned}
$$

From Markov inequality, for every $\varepsilon>0$, we have

$$
P\left[\frac{1}{k_{n}} \log \mathcal{L}_{n}(\omega) \geq \varepsilon\right]=P\left[\mathcal{L}_{n}(\omega) \geq \exp \left(k_{n} \varepsilon\right)\right] \leq 1 \cdot \exp \left(-k_{n} \varepsilon\right) .
$$


Hence

$$
\sum_{n=1}^{\infty} P\left[\frac{1}{k_{n}} \log \mathcal{L}_{n}(\omega) \geq \varepsilon\right] \leq \sum_{n=1}^{\infty} \exp \left(-k_{n} \varepsilon\right)<\infty
$$

By Borel-Cantelli lemma, we have

$$
P\left[\limsup \frac{1}{k_{n}} \log \mathcal{L}_{n}(\omega) \geq 2 \varepsilon\right]=0,
$$

for any $\varepsilon>0$, (1.3) follows immediately.

\section{Main results and proofs}

Theorem 1. Let $\left(X_{n}\right)_{n \in \mathbf{N}}, \mathcal{L}_{n}(\omega), \mathcal{L}(\omega)$ be defined as above, $\left(B_{n}\right)_{n \in \mathbf{N}}$ be a sequence of Borel sets of the real line. Let $S_{n, k_{n}}(\omega)=\frac{1}{k_{n}} \sum_{j=n}^{n+k_{n}-1} \mathbf{1}_{B_{j}}\left(X_{j}\right)$, and assume

$$
c=\limsup _{n} \frac{1}{k_{n}} \sum_{j=n}^{n+k_{n}-1} P\left(X_{j} \in B_{j}\right)
$$

then

$$
\limsup _{n} S_{n, k_{n}}(\omega) \leq(\sqrt{\mathcal{L}(\omega)}+\sqrt{c})^{2}, \text { a.e. }
$$

where $\mathbf{1}_{B_{n}}(\cdot)$ be the indicator function of $B_{n}$.

Proof. Assume $s>0$ to be a constant, and let

$$
h_{j}\left(x_{j}\right)=\frac{s^{\mathbf{1}_{B_{j}}\left(x_{j}\right)} f_{j}\left(x_{j}\right)}{1+(s-1) \int_{B_{j}} f_{j}\left(x_{j}\right) d x_{j}}, \quad j=1,2, \ldots
$$

It is not difficult to see that $\int h_{j}\left(x_{j}\right) d x_{j}=1, j=1,2, \ldots$ Let

$$
\Lambda_{n}(s, \omega)= \begin{cases}\frac{\Pi_{j=n}^{n+k_{n}-1} h_{j}\left(X_{j}\right)}{g^{n, n+k_{n}-1}\left(X_{n, \ldots,} X_{n+k_{n}-1}\right)}, & \text { if denominator }>0 \\ 0, & \text { otherwise }\end{cases}
$$

From Lemma 1 , there exists $A(s) \in \mathcal{F}, P(A(s))=1$, such that

$$
\underset{n}{\lim \sup } \frac{1}{k_{n}} \log \Lambda_{n}(s, \omega) \leq 0, \quad \omega \in A(s)
$$

Since $\int_{B_{j}} f_{j}\left(x_{j}\right) d x_{j}=P\left(X_{j} \in B_{j}\right)$, by (2.3) we have

$$
\begin{aligned}
& \prod_{j=n}^{n+k_{n}-1} h_{j}\left(x_{j}\right) \\
= & \prod_{j=n}^{n+k_{n}-1} \frac{s^{\mathbf{1}_{B_{j}}\left(x_{j}\right)} f_{j}\left(x_{j}\right)}{1+(s-1) \int_{B_{j}} f_{j}\left(x_{j}\right) d x_{j}} \\
= & s^{\sum_{j=n}^{n+k_{n}-1} \mathbf{1}_{B_{j}}\left(x_{j}\right)} \prod_{j=n}^{n+k_{n}-1} \frac{f_{j}\left(x_{j}\right)}{1+(s-1) P\left(X_{j} \in B_{j}\right)}
\end{aligned}
$$


It follows from (1.1), (2.4) and (2.6) that

$$
\log \Lambda_{n}(s, \omega)=\sum_{j=n}^{n+k_{n}-1} \mathbf{1}_{B_{j}}\left(X_{j}\right) \log s-\sum_{j=n}^{n+k_{n}-1} \log \left[1+(s-1) P\left(X_{j} \in B_{j}\right)\right]+\log \mathcal{L}_{n}(\omega)
$$

(2.5) and (2.7) yield

$$
\underset{n}{\limsup } \frac{1}{k_{n}}\left(\log s \sum_{j=n}^{n+k_{n}-1} \mathbf{1}_{B_{j}}\left(X_{j}\right)-\sum_{j=n}^{n+k_{n}-1} \log \left[1+(s-1) P\left(X_{j} \in B_{j}\right)\right]+\log \mathcal{L}_{n}(\omega)\right) \leq 0, \quad \omega \in A(s)
$$

Let $s>1$, dividing the two sides of (2.8) by $\log s$, we have

$$
\underset{n}{\limsup } \frac{1}{k_{n}}\left(\sum_{j=n}^{n+k_{n}-1} \mathbf{1}_{B_{j}}\left(X_{j}\right)-\sum_{j=n}^{n+k_{n}-1} \frac{\log \left[1+(s-1) P\left(X_{j} \in B_{j}\right)\right]}{\log s}+\frac{\log \mathcal{L}_{n}(\omega)}{\log s}\right) \leq 0, \quad \omega \in A(s)
$$

By (1.2), (2.9) and the property $\lim \sup _{n}\left(a_{n}-b_{n}\right) \leq d \Rightarrow \lim \sup _{n}\left(a_{n}-c_{n}\right) \leq \lim \sup _{n}$ $\left(b_{n}-c_{n}\right)+d$, one gets

$$
\limsup _{n} \frac{1}{k_{n}}\left(\sum_{j=n}^{n+k_{n}-1} \mathbf{1}_{B_{j}}\left(X_{j}\right)-\sum_{j=n}^{n+k_{n}-1} \frac{\log \left[1+(s-1) P\left(X_{j} \in B_{j}\right)\right]}{\log s}\right) \leq \frac{\mathcal{L}(\omega)}{\log s}, \quad \omega \in A(s)
$$

By (2.10) and the property of the superior above and the inequality $0<\log (1+x) \leq x$ $(x>0)$, we obtain

$$
\begin{aligned}
& \limsup _{n} S_{n, k_{n}}(\omega) \\
& \leq \underset{n}{\limsup } \frac{1}{k_{n}}\left(\sum_{j=n}^{n+k_{n}-1} \frac{\log \left[1+(s-1) P\left(X_{j} \in B_{j}\right)\right]}{\log s}\right)+\frac{\mathcal{L}(\omega)}{\log s} \\
& \leq \limsup _{n} \frac{1}{k_{n}}\left(\sum_{j=n}^{n+k_{n}-1} \frac{(s-1) P\left(X_{j} \in B_{j}\right)}{\log s}\right)+\frac{\mathcal{L}(\omega)}{\log s} \\
& \leq c\left(\frac{s-1}{\log s}\right)+\frac{\mathcal{L}(\omega)}{\log s}, \quad \omega \in A(s)
\end{aligned}
$$

(2.11) and the inequality $1-\frac{1}{s}<\log s(s>1)$ imply

$$
\limsup _{n} S_{n, k_{n}}(\omega) \leq c \cdot s+\frac{s \mathcal{L}(\omega)}{s-1}, \quad \omega \in A(s)
$$

Let $D$ be a set of countable real numbers dense in the interval $(1,+\infty)$, and let $A^{*}=$ $\mathrm{n}_{s \in D} A(s), g(s, x)=c s+s x /(s-1)$, then we have by $(2.12)$

$$
\limsup _{n} S_{n, k_{n}}(\omega) \leq g(s, \mathcal{L}(\omega)), \quad \omega \in A^{*}, \quad s \in D
$$

Let $c>0$, it easy to see that if $\mathcal{L}(\omega)>0$, a.e., then, for fixed $\omega, g(s, \mathcal{L}(\omega))$ as a function of $s$ attains its smallest value $g(1+\sqrt{\mathcal{L}(\omega) / c}, \mathcal{L}(\omega))=2 \sqrt{c \mathcal{L}(\omega)}+\mathcal{L}(\omega)+c$ on the interval $(1,+\infty)$, and $g(s, 0)$ is increasing on the interval $(1,+\infty)$ and $\lim _{s \rightarrow 1}+g$ $(s, 0)=0$. For each $\omega \in A^{*} \cap A(1)$, if $\mathcal{L}(\omega) \neq \infty$, take $\kappa_{n}(\omega) \in D, n=1,2, \ldots$, such that $\kappa_{n}(\omega) \rightarrow 1+\sqrt{\mathcal{L}(\omega) / \mathcal{c}}$. We have by the continuity of $g(s, \mathcal{L}(\omega))$ with respect to $s$, 


$$
\lim _{n \rightarrow+\infty} g\left(\kappa_{n}(\omega), \mathcal{L}(\omega)\right)=(\sqrt{\mathcal{L}(\omega)}+\sqrt{c})^{2}
$$

By (2.13), we obtain

$$
\limsup S_{n, k_{n}} \leq g\left(\kappa_{n}(\omega), \mathcal{L}(\omega)\right), \quad n=1,2, \ldots
$$

(2.14) and (2.15) imply

$$
\limsup _{n} S_{n, k_{n}}(\omega) \leq(\sqrt{\mathcal{L}(\omega)}+\sqrt{c})^{2}, \quad \omega \in A^{*} \cap A(1)
$$

If $\mathcal{L}(\omega)=\infty,(2.16)$ holds trivially. Since $P\left(A^{*} \cap A(1)\right)=1,(2.2)$ holds by (2.16), when $c>0$.

When $c=0$, we have by letting $s=e$ in (2.11),

$$
\limsup _{n} S_{n, k_{n}}(\omega) \leq \mathcal{L}(\omega), \quad \omega \in A(e)
$$

since $P(A(e))=1,(2.2)$ also holds by (2.17) when $c=0$. $\square$

Theorem 2. Let $\left(X_{n}\right)_{n \in \mathbf{N}}, \mathcal{L}_{n}(\omega), \mathcal{L}(\omega),\left(B_{n}\right)_{n \in \mathbf{N}}, S_{n, k_{n}}(\omega)$ be defined as in Theorem 1 and assume

$$
c^{\prime}=\liminf _{n} \frac{1}{k_{n}} \sum_{j=n}^{n+k_{n}-1} P\left(X_{j} \in B_{j}\right)
$$

then, if $0 \leq \mathcal{L}(\omega) \leq c^{\prime}$ a.e., then

$$
\liminf _{n} S_{n, k_{n}}(\omega) \geq c^{\prime}-2 \sqrt{c^{\prime} \mathcal{L}(\omega), \quad \text { a.e. }}
$$

Proof. Let $0<s<1$, dividing the two sides of (2.8) by $\log s$, we have

$$
\liminf _{n} \frac{1}{k_{n}}\left(\sum_{j=n}^{n+k_{n}-1} \mathbf{1}_{B_{j}}\left(X_{j}\right)-\sum_{j=n}^{n+k_{n}-1} \frac{\log \left[1+(s-1) P\left(X_{j} \in B_{j}\right)\right]}{\log s}+\frac{\log \mathcal{L}_{n}(\omega)}{\log s}\right) \geq 0, \quad \omega \in A(s)
$$

By (1.2), (2.20) and the property $\lim \inf _{n}\left(a_{n}-b_{n}\right) \geq d \Rightarrow \lim \inf _{n}\left(a_{n}-c_{n}\right) \geq \lim \inf _{n}\left(b_{n}\right.$ $\left.-c_{n}\right)+d$, one gets

$$
\liminf _{n} \frac{1}{k_{n}}\left(\sum_{j=n}^{n+k_{n}-1} \mathbf{1}_{B_{j}}\left(X_{j}\right)-\sum_{j=n}^{n+k_{n}-1} \frac{\log \left[1+(s-1) P\left(X_{j} \in B_{j}\right)\right]}{\log s}\right) \geq \frac{\mathcal{L}(\omega)}{\log s}, \quad \omega \in A(s)
$$

By (2.21) and the property of the inferior above and the inequality $\log (1+x) \leq x(-1$ $<x \leq 0$ ), we obtain

$$
\begin{aligned}
& \liminf _{n} S_{n, k_{n}}(\omega) \\
& \geq \liminf _{n} \frac{1}{k_{n}}\left(\sum_{j=n}^{n+k_{n}-1} \frac{\log \left[1+(s-1) P\left(X_{j} \in B_{j}\right)\right]}{\log s}\right)+\frac{\mathcal{L}(\omega)}{\log s} \\
& \geq \liminf _{n} \frac{1}{k_{n}}\left(\sum_{j=n}^{n+k_{n}-1} \frac{(s-1) P\left(X_{j} \in B_{j}\right)}{\log s}\right)+\frac{\mathcal{L}(\omega)}{\log s} \\
& \geq c^{\prime}\left(\frac{s-1}{\log s}\right)+\frac{\mathcal{L}(\omega)}{\log s}, \quad \omega \in A(s)
\end{aligned}
$$


(2.22) and the inequality $1-\frac{1}{s}<\log s$ and $\log s<s-1(0<s<1)$ imply

$$
\liminf _{n} S_{n, k_{n}}(\omega) \geq c^{\prime} \cdot s+\frac{\mathcal{L}(\omega)}{s-1}, \quad \omega \in A(s) \cap A(1)
$$

Let $D^{\prime}$ be a set of countable real numbers dense in the interval $(0,1)$, and let $A_{*}=\cap_{s \in D^{\prime}} A(s), h(s, x)=c^{\prime} s+x /(s-1)$, then we have by $(2.23)$

$$
\liminf _{n} S_{n, k_{n}}(\omega) \geq h(s, \mathcal{L}(\omega)), \quad \omega \in A_{* \prime} \quad s \in D^{\prime}
$$

Let $c^{\prime}>0$, it easy to see that if $0<\mathcal{L}(\omega)<c^{\prime}$, a.e., then, for fixed $\omega, h(s, \mathcal{L}(\omega))$ as a function of $s$ attains its maximum value $h\left(1-\sqrt{\mathcal{L}(\omega) / c^{\prime}}, \mathcal{L}(\omega)\right)=c^{\prime}-2 \sqrt{c^{\prime} \mathcal{L}(\omega)}$, on the interval $(0,1)$, and $h(s, 0)$ is increasing on the interval $(0,1)$ and $\lim _{s \rightarrow 1}+h(s, 0)=$ $c^{\prime}$. For each $\omega \in A * \cap A(1)$, if $\mathcal{L}(\omega) \neq \infty$, take $l_{n}(\omega) \in D^{\prime}, n=1,2, \ldots$, such that $l_{n}(\omega) \rightarrow 1-\sqrt{\mathcal{L}(\omega) / c^{\prime}}$. We have by the continuity of $h(s, \mathcal{L}(\omega))$ with respect to $s$,

$$
\lim _{n \rightarrow+\infty} h\left(l_{n}(\omega), \mathcal{L}(\omega)\right)=c^{\prime}-2 \sqrt{c^{\prime} \mathcal{L}(\omega)}
$$

By (2.24), we obtain

$$
\liminf _{n} S_{n, k_{n}} \geq h\left(l_{n}(\omega), \mathcal{L}(\omega)\right), \quad n=1,2, \ldots
$$

(2.25) and (2.26) imply

$$
\liminf _{n} S_{n, k_{n}}(\omega) \geq c^{\prime}-2 \sqrt{c^{\prime} \mathcal{L}(\omega)}, \quad \omega \in A_{*} \cap A(1)
$$

If $\mathcal{L}(\omega)=\infty,(2.27)$ holds trivially. Since $P(A * \cap A(1))=1$, (2.19) holds by (2.27), when $c^{\prime}>0$. (2.19) also holds trivially when $c^{\prime}=0$. $\square$

Remark 2. In case $\mathcal{L}(\omega)>c^{\prime} \geq 0$, a.e., we cannot get a better lower bound of $\lim \inf _{n} S_{n, k_{n}}(\omega)$. This motivates the following problem: under the conditions of Theorem 2, how to get a better lower bound of $\liminf _{n} S_{n, k_{n}}(\omega)$ in case of $\mathcal{L}(\omega)>c^{\prime} \geq 0$, a.e.?

Definition 2. (Generalized empirical distribution function) Let $\left(X_{n}\right)_{n \in \mathbf{N}}$ be identically distribution with common distribution function $F$, for each $m, n \in \mathbf{N}$, let

$$
F_{m, n}(x)=\frac{1}{n} \sum_{k=m}^{m+n-1} \mathbf{1}_{\left(X_{k} \leq x\right)}
$$

$F_{m, n}=$ the observed frequency of values that are $\leq x$ from time $m$ to $m+n-1$. The $F_{1, n}$ is the usual empirical distribution function, hence the name given above.

In particular, let $B=(-\infty, x], x \in R$ in Theorems 1 and 2 , we can get a strong limit theorem for the generalized empirical distribution function.

Corollary 1. Let $\left(X_{n}\right)_{n \in \mathbf{N}}$ be i.i.d. random variables with common distribution function $F$, let $B_{n}=(-\infty, x], n=1,2, \ldots$, then

$$
\lim _{n} F_{n, n+k_{n}-1}(x)=F(x) \text {, a.e. }
$$

Corollary 2. Let $\left(X_{n}\right)_{n \in \mathbf{N}}$ be independent random variables and $\left(B_{n}\right)_{n \in \mathbf{N}}$ be as Theorem 1 , then 


$$
\lim _{n} \frac{1}{k_{n}} \sum_{j=n}^{n+k_{n}-1}\left[\mathbf{1}_{B_{j}}\left(X_{j}\right)-P\left(X_{j} \in B_{j}\right)\right]=0 \text {, a.e. }
$$

Proof. Note that $P\left(X_{j} \in B_{j}\right) \leq 1, j=1,2, \ldots$ and in this case, $0 \leq c, c^{\prime} \leq 1, \mathcal{L}(\omega)=0$ a. e., we have by $(2.11)$

$$
\underset{n}{\limsup } \frac{1}{k_{n}}\left(\sum_{j=n}^{n+k_{n}-1} \mathbf{1}_{B_{j}}\left(X_{j}\right)-\sum_{j=n}^{n+k_{n}-1} \frac{\log \left[1+(s-1) P\left(X_{j} \in B_{j}\right)\right]}{\log s}\right) \leq 0, \quad \omega \in A(s)
$$

by (2.29) and the property of the superior above and the inequality $0 \leq \log (1+x) \leq x$ $(x>0)$, we obtain

$$
\begin{aligned}
& \limsup _{n} \frac{1}{k_{n}} \sum_{j=n}^{n+k_{n}-1}\left[\mathbf{1}_{B_{j}}\left(X_{j}\right)-P\left(X_{j} \in B_{j}\right)\right] \\
& \leq \underset{n}{\limsup } \frac{1}{k_{n}}\left(\sum_{j=n}^{n+k_{n}-1} \frac{\log \left[1+(s-1) P\left(X_{j} \in B_{j}\right)\right]}{\log s}-P\left(X_{j} \in B_{j}\right)\right) \\
& \leq \underset{n}{\limsup } \frac{1}{k_{n}}\left(\sum_{j=n}^{n+k_{n}-1} \frac{(s-1) P\left(X_{j} \in B_{j}\right)}{\log s}-P\left(X_{j} \in B_{j}\right)\right) \\
& \leq\left(\frac{s-1}{\log s}-1\right), \quad \omega \in A(s)
\end{aligned}
$$

Analogously as in the proof of Theorem 1, we obtain

$$
\limsup _{n} \frac{1}{k_{n}} \sum_{j=n}^{n+k_{n}-1}\left[\mathbf{1}_{B_{j}}\left(X_{j}\right)-P\left(X_{j} \in B_{j}\right)\right] \leq 0 \text {, a.e. }
$$

Similarly, we have $\liminf _{n} \frac{1}{k_{n}} \sum_{j=n}^{n+k_{n}-1}\left[1_{B_{j}}\left(X_{j}\right)-P\left(X_{j} \in B_{j}\right)\right] \geq 0$, a.e. hence (2.28) follows immediately.

Remark 3. Let $B_{n}=B$, Corollary 2 implies that $\frac{\lim S_{n, k_{n}}}{k_{n}}=P\left(X_{1} \in B\right)$ which gives the strong law of large numbers for the delayed arithmatic means.

\section{Acknowledgements}

This work is supported by The National Natural Science Foundation of China (Grant No. 11071104) and the An Hui University of Technology research grant: D2011025. The authors would like to thank two referees for their insightful comments which resulted in improving Theorems 1, 2 and Corollary 2 significantly.

\section{Author details}

${ }^{1}$ Department of Mathematics \& Physics, HeFei University, HeFei 230601, P. R. China ${ }^{2}$ Faculty of Mathematics \& Physics, AnHui University of Technology, Ma'anshan 243002, P. R. China

\section{Authors' contributions}

WZ and DF carried out the design of the study and performed the analysis. DF drafted the manuscript. All authors read and approved the final manuscript.

\section{Competing interests}

The authors declare that they have no competing interests.

Received: 29 October 2011 Accepted: 31 May 2012 Published: 31 May 2012

\section{References}

1. Zygmund, A: Trigonometric Series 1. Cambridge Universitiy Press, Cambridge (1959) 
2. Chow, YS: Delayed sums and Borel summability for independent, identically distributed random variables. Bull Inst Math Academia Sinica. 1, 207-220 (1972)

3. Lai, TL: Limit theorems for delayed sums. Ann Probab. 2(3), 432-440 (1974). doi:10.1214/aop/1176996658

4. Chen, PY: Limiting behavior of delayed sums under a non-identically distribution setup. Ann Braz Acad Sci. 80(4), 617-625 (2008)

5. Liu, W: Strong deviation theorems and analytical method. Academic press, Beijing (2003) (in Chinese)

doi:10.1186/1029-242X-2012-124

Cite this article as: Fang-qing and Zhong-zhi: Limit theorems for delayed sums of random sequence. Journal of Inequalities and Applications 2012 2012:124.

Submit your manuscript to a SpringerOpen ${ }^{\circ}$ journal and benefit from:

- Convenient online submission

- Rigorous peer review

- Immediate publication on acceptance

- Open access: articles freely available online

- High visibility within the field

- Retaining the copyright to your article

Submit your next manuscript at $\boldsymbol{s p r i n g e r o p e n . c o m ~}$ 